\title{
12
}

\section{IMMERSIVE GAMING AS JOURNALISM}

\author{
Jonne Arjoranta, Raine Koskimaa, and Marko Siitonen
}

As a field, journalism constantly strives to connect with its audiences and find ways to utilize emerging media technologies in its operations. Sometimes this is done simply to reach audiences that have ceased to use traditional media, or to invite new audiences in, but often it is also a matter of perceived benefits related to using the affordances of certain technological solutions. One recent example is the interest surrounding the concept of immersion. For example, recent research has explored the question of whether there is a connection between the immersiveness of a technology and the users' empathetic responses (Archer \& Finger 2018; Herrera et al. 2018). While some of these explorations are done specifically within the context of journalism, there is a considerable amount of overlap between different fields of interest, warranting a broader look at how the concept of immersion has been theorized.

This chapter discusses immersion and how it may be applied to journalism. In order to do so, we start by unraveling the concept of immersion itself as a reminder that it is not only connected to virtual reality (VR) or augmented reality (AR) technologies, but rather that it is a multifaceted concept that may be understood in many ways. Here, we turn specifically to the theorizing done in the context of digital games research, where immersion and some related concepts have been explored in detail over the last decades. This theorizing helps us see how immersion can be understood and in what way the concept may be problematic. The chapter maps out some of the historical precedents for immersion, discusses alternative and related concepts (such as presence), and how this understanding may be used to inform discussions of immersive journalism.

\section{The magic circle of immersion}

Immersion is a recurring concept when discussing video games and the way that players engage with them. It is often seen as a given that players immerse themselves 
in the game when playing. The roots of the concept of immersion related to play can be traced back to the classic Homo Ludens (1971[1938]) by Johan Huizinga. He never used the word "immersion", but his concept of the "magic circle" can be seen as the precursor for most game immersion discourse. According to Huizinga, a player is transferred from the everyday reality to the realm of game for the duration of play. While within the magic circle, the rules of play replace the everyday setting, and the strong sensation of the game space characterizes play: "[...] in this intensity, this absorption, this power of maddening, lies the very essence, the primordial quality of play" (Huizinga 1971[1938], 2).

The concept of the magic circle has been adopted by most of the game and play theorists since Huizinga, but, as there is not a very systematic definition of the concept in Homo Ludens, it has received numerous interpretations. For many game scholars today, the concept of the magic circle actually refers primarily to Katie Salen and Eric Zimmerman's characterization, which owes to Huizinga but cannot be fully reduced to it. They use the concept as a "shorthand for the idea of a special place in time and space created by a game" and to describe "where the game takes place" (Salen \& Zimmerman 2004, 95).

Jaakko Stenros (2014) has gone through the history and uses of the magic circle, and found three main interpretations: 1) spatial or "arena", 2) social contract, and 3) psychological bubble. Of these three, the psychological bubble, "the "protective frame' that surrounds a person in a playful state of mind", is most directly related to immersion. Drawing from Michael J. Apter's (1991, 14-15) reversal theory of personality, motivation and emotion, which recognizes opposite metamotivational states of "serious" and "playful", Stenros concludes that when a player is within the psychological bubble, “[t]here is a 'border' around her experience that guides her interpretation of the situation" (2014, 173-174).

Another influence on conceptualizing immersion in games comes from Janet Murray's book Hamlet in the Holodeck (1997), even though the book itself focused on issues related to virtual reality and future narrative forms. Murray has a different approach than Huizinga, who was focusing on the characteristics of play as a specific type of activity. Murray starts from the power of narration to transfer the audience to another world created by the narrative. She sees online and virtual worlds as the latest phase in the development of narrative media, and replaces such concepts as "make-believe" and "willing suspension of disbelief", more familiar in literary studies, with the concept of immersion adopted in the then-novel field of computer-based virtual worlds:

Immersion is a metaphorical term derived from the physical experience of being submerged in water. We seek the same feeling from a psychologically immersive experience [...] the sensation of being surrounded by a completely other reality [...] that takes over all of our attention, our whole perceptual apparatus. 
François Laramée, when writing on game design principles, neatly illustrates this kind of approach to immersion, and promotes it as the highest priority of all entertainment: "All forms of entertainment strive to create suspension of disbelief, a state in which the player's mind forgets that it is being subjected to entertainment and instead accepts what is perceived as reality" (Laramée 2002, according to Salen \& Zimmerman 2004, 450). It is, however, quite problematic to assume immersion requires the "forgetting of the reality". Instead, the phenomenological concept of bracketing, temporarily setting aside the assumed objective reality, would be a more accurate expression here. Other examples of immersion-characterized video game theory can be found from James Newman, "videogames may be characterized by a sense of 'being there"” $(2004,17)$, or from Nick Yee (2002), who identified one of the core factors for MMORPGs' (massively multiplayer online role-playing games') holding power as being "the immersive nature of these virtual environments" (9).

\section{Expanding the concept of immersion}

The way Murray (1997) conceptualizes immersion emphasizes fictional content in the process. When applied to games and play, this is problematic in two obvious ways: 1) there are games, also highly popular ones, without fictional world or narrative content, and 2) games and game play involve other aspects than the fictional game world, also in those cases where such a world exists. A player of Tetris, for example, may be clearly "immersed" in the game action, even if there is not an apparent fictional world to speak of. It is necessary to distinguish the two levels of videogame play: the represented world or diegetic level (which may be a narrative-based fictional world, or an abstract world) and the real-life player action, non-diegetic level, in which the player operates the game controller and devises her strategies. Alison McMahan (2003) has noted how immersion may take place in regards to both of these levels. For McMahan, immersion refers both to how the player may be "caught up in the world of the game's story", but also to her "love of the game and the strategy that goes into it" $(2003,68)$.

According to McMahan (2003), the use of immersion in game studies has suffered from serious confusion, partly caused by the borrowing of terms from the fields of virtual reality and interface design. She proposes presence as a more general, and in many cases more accurate, term than immersion. McMahan defines presence as "the artificial sense that a user has in a virtual environment that the environment is unmediated". Sense of presence is a complex phenomenon involving a set of dimensions: quality of social interaction, realism in the environment, the effect of "transportation", immersiveness generated by the interface, the user's ability to accomplish significant actions within the environment, and users responding to the computer as an intelligent, social agent. The environments and experiences vary greatly depending on the presence or absence of, and interplay between, these dimensions, but all of the six dimensions share the perceptual illusion of nonmediation (McMahan 72-73, referring to Lombard \& Ditton). 
Immersion and engagement are both aspects of presence in McMahan's framework. Immersion relates to presence on a diegetic level, whereas engagement relates to presence on a non-diegetic level, including such actions as gaining points and devising a winning strategy, etc. (McMahan 2003, 69, 79). In this framework, immersion is just one dimension of presence, and much of immersion talk actually relates to presence instead.

For example, in the game 1979 Revolution: Black Friday (iNK Stories 2016) the player adopts the role of Reza Shirazi, a photojournalist present in the 1979 Iranian revolution. One of the central ways of interacting with the game is taking pictures of the events happening around Reza. The photos taken by the player are then contrasted with historical pictures taken by Michel Setboun, showing what the events looked like outside of the game. This necessarily reminds the player of events beyond the game, lessening the player's immersion - but the reminder that the events they are witnessing are based on historical events may also enhance their sense of engagement with the game. From a journalistic point of view, the inclusion of authentic photos is easier to defend than using only imaginary examples. Therefore, at least in this case, pursuing immersion alone would not lead to the optimal outcome.

Salen and Zimmerman's (2004) interpretation of the possibility of shifting frames is especially valuable in that it is able to explain how frequent shifts between immersion in the game world and engagement in the game play may take place so smoothly, by their both being encapsulated within the magic circle (ibid., 455). The presence in the magic circle does not break, despite movement between the states of immersion and engagement. It is, for example, quite common for the person involved in the game play to momentarily acknowledge also the space outside the magic circle, be it one's own living room, an eSports arena, or a public transportation vehicle, so that the periods of deep immersion and engagement may be relatively short, but also returning to the magic circle may happen quickly again. Of course, in the case of virtual reality specifically, it may be that the technologyrelated issues such as physical visors and earphones, or long loading times for software, prohibit such behavior, and push users towards spending a longer time within the VR experience.

Finally, game theorists have tried to adopt the concept of immersion to better suit interactive media. Calleja $(2011,1)$ argues that the concepts of presence and immersion both assume that the relation between a user and a system is unidirectional, from a physical reality into a virtual one, or a "dive of human subjectivity into a containing vessel". This metaphor does not work in media where the user can affect their surroundings, because it does not sufficiently take into account the medium's role. He suggests that, instead, a better metaphor would be incorporation, the absorption of a virtual environment into consciousness, yielding a sense of habitation, which is supported by the systemically upheld embodiment of the player in a single location represented by the avatar.

This goes beyond immersion, since it includes how the system acknowledges the player's existence. This does not automatically mean that incorporation is a better 
concept for understanding immersive experiences, but it is a useful reminder that virtual realities can have varying amounts of interactivity with the users.

\section{Sensory immersion and VR technology}

A somewhat different take on immersion is provided by Frans Mäyrä, who brings the choice of visual perspective into the game as an additional factor. Strong sensory immersion is provided, especially by the first-person view in games played on screen, and even more so with VR headsets. Freedom of moving around and instantaneous feedback from the game environment give rise to immersion in actions of play, which can be called a "challenge-based form of immersion into games" (2008, 107-108.) This approximately resembles McMahan's engagement, but also comes close to Mihaly Csikszentmihalyi’s (Csikszentmihalyi \& Csikszentmihalyi 1975) concept of flow. Mäyrä also recognizes immersion in the game world, describing it as absorption: "[...] another kind of immersion involved, as the player becomes emotionally as well as intellectually absorbed in the game world. [...] we can call it imaginative immersion" (ibid., 109). If we compare McMahan and Mäyrä with each other, the models are quite compatible, with the exception that sensory immersion is of a different conceptual level than presence. Sensory immersion is clearly one way of creating a sense of presence, but not the only one available; we can find immersion and engagement, for example, in interactive fiction (text-based adventure games).

When looking at VR immersion specifically, there are certain aspects of the presented world which contribute to immersion, in addition to the sensory apparatus. The first-person perspective fosters immersion, but what Newman (2004) has called "first-hand participation" may be an even more significant factor, meaning active agency in the game world. First-hand participation is not reliant upon firstperson perspective, but may appear independently and can "engender a degree of interactive connection with the gameworld that goes far beyond the abstracted 'use' of a system or vicarious identification with and manipulation of an iconic character or world" $(2004,142)$. It is important here that first-hand participation is conceptually separated from the game character (avatar), as they are usually strongly limited in gestural affordances when compared to degrees of freedom when using one's own body.

The oft-cited early example of building VR immersion is Hunger in Los Angeles (2012), a VR experiment recreating a crisis in a food bank line in Los Angeles. The experiment, which combined a 3D-modeled environment with an audio recording of the actual incident, aimed at recreating the feeling of "being there", according to the head developer Nonny de la Peña. While the technology of the time consisted of prototypes, there was a clear intention by the designers to recreate reality as faithfully as possible. The characters, the world, and especially the audio track point to a "real" reality which is experienced via a first-person perspective. However, there is little real first-hand participation, due to the fact that the player is represented by a bodiless floating "ghost", and the event will play out the same way regardless of their actions. 
Similarly, in CNN's An ordinary day in North Korea, the camera is placed so high as to break the illusion of human viewpoint. In other typical cases, such as the BBC's story on weapons-training in Polish schools, the focus of the story is on a specific person, who presents a focal point, but here again the reader finds themselves in the role of a faceless observer without any other agency than to turn around. This might be an intentional design choice: by limiting the user's agency, CNN and the BBC have tighter editorial control on what their VR experiences convey to their users. Most likely it also speaks of the technical limitations of the camera setups used to construct most VR journalistic pieces: after all, the procedure of recording VR footage often includes using static camera(s) and trying to make sure the camera crew are not visible in the captured footage.

Whatever the reasons may be, it is clear that most contemporary examples of VR journalism have emphasized audiovisual fidelity or sensory immersion. From a game studies point of view this appears problematic. In a somewhat polemical fashion, Katie Salen and Eric Zimmerman (2004) call the over-emphasis of sensory immersion "the immersive fallacy":

[...] the idea that the pleasure of a media experience lies in its ability to sensually transport the participant into an illusory, simulated reality. [...] Although the immersive fallacy has taken hold in many fields, it is particularly prevalent in the digital game industry.

Salen E Zimmerman 2004, 450-451

The mistake behind immersive fallacy is to focus solely on the representational aspect and neglect the interface issues and play activity itself. As Marie-Laure Ryan has noted, in many fields over the past decades immersion has become less important and self-referentiality rendering the medium visible has gained more ground (Ryan 2001, 349). Thanks to this cultural movement, other media are largely avoiding the immersive fallacy, whereas, according to Salen and Zimmerman, "within the digital game industry, belief in the immersive fallacy remains alive and well" $(2004,451)$. The immersive fallacy does not mean that there would not be immersion related to games and other media at all, but rather that immersion usually appears as an element within a complex process of mediation. It is prudent to ask whether there is a distinct risk that the field of journalism, in the pursuit of exploring emerging technologies' affordances, is also in danger of falling into the trap of immersive fallacy by considering immersion in too narrow a way?

\section{A gameful approach to immersive journalism}

What would it mean in practice if there were to be more interaction and more intense gamification added to VR journalism? What kind of consequences would it entail in journalistic terms, and what would it be like as an audience experience? It 
is hard to avoid the notion that interactivity and gamification will definitely make things more complicated. The main challenge that all interactive storytelling faces is finding a balance between a scripted story, designed in advance, and the user's freedom of choice and first-hand participation. The more freedom is granted to the user, the more difficult it is to deliver a specific message with a fixed perspective. Game designers, however, have come up with several techniques to accomplish this feat. Often the trick involves making the player believe they have more freedom of choice in the game world than they actually possess. Since there is a lack of truly interactive, gamified pieces of VR journalism to date, we will next use newsgames as examples of journalistic content where game design principles emphasizing firsthand participation and immediate feedback are employed, giving rise to immersive and engaging experiences.

For example, The Uber Game (Financial Times 2017) sees the player take on the role of an Uber driver. The player has to choose how to approach the job, highlighting choices such as which kind of car to get (Uber drivers have to provide their own cars) and whether to get a business license or not. The game shows that the question of whether one can make it in the "gig economy" is often a matter of chance and that it may involve sacrificing other, valuable things, such as family time, in order to chase the elusive bonuses offered by the company. The picture portrayed by the game is very different from the one advertised by the company, which highlights the drivers' freedom to work as they see fit. A game system like The Uber Game is well fit to portray systemic phenomena, such as the complex reality of handling a job in the gig economy. To an extent, other journalistic media can also portray experiences of precariousness, but games are perhaps uniquely positioned to reflect on the relation between choice and chance, freedom and uncertainty. Players can try to inhabit the position portrayed by the game themselves, gaining at least some insight into the uncertainty of the situation.

If the goal is to create gameful journalism, there are new issues that must be taken into account. The tools to create these experiences are quite developed, but far from trivial to use. Designing gameful journalism takes a skillset that may not necessarily be obtained by doing traditional journalism. Even if gameful journalism promises to be able to do some things better than some other journalistic approaches, this only applies if the approach is used successfully. This takes resources, skills, and reflection on the ethical dimensions.

\section{Conclusion}

If we apply these lessons from how game studies have approached immersion to how immersion may be pursued or utilized in the field of journalism, we can see some suggestions for what to focus on and what to avoid. First, it is necessary to begin with a reminder that there is no one sense of immersion. Rather, the word has been used in different ways in different contexts. They are all more or less compatible, but highlight different aspects of the experience. Here we have drawn, for example, from McMahan (2003), who proposed a distinction between presence, 
engagement, and immersion, where presence operates as an umbrella term under which a more content-driven immersion and a more user-action-centered engagement are situated. This kind of division is useful as it reminds us that while speaking about immersion it may actually be engagement that technologically oriented developers are after - and that, if this division is not clear, it may lead to misunderstanding the available affordances, as well as their possible effects. It also reminds us that one does not necessarily need high-quality graphics or realistic virtual environments to be immersed in journalistic storytelling.

It should be clear by now that different applications, be they virtual worlds or games, induce different types of immersion (Ermi \& Mäyrä 2005). It is also worth noting that different types of immersion may give rise to different ethical requirements (Mäyrä 2008, 125). For example, the question of whether or not to include the "shadow of a leggy tripod with a spherical camera head" in the VR recording becomes not only technical but also ethical when one considers the repercussions of "hiding" authorship and the constructed nature of VR news stories (Kool 2016, 9). Indeed, for a few years now there have been calls for increased transparency regarding VR journalism. As Tom Kent put it in his essay, "Viewers need to know how VR producers expect their work to be perceived, what's been done to guarantee authenticity and what part of a production may be, frankly, supposition" (2015 n.p.).

As the question of immersion is closely tied to technological affordances, it is also worthwhile to keep a close eye on developments that take place outside of and parallel to VR. The concept of Augmented or Added Reality, AR, is often used together with VR, and they are considered as close relatives. In some aspects this is true, but it is especially in regards to immersion that their premises should be closely scrutinized. AR applications posit features on top of the physical surroundings. This may take the form of extra information layers on the perceived environment, and the power of AR lies in incorporating the familiar physical environment into the partially crafted experience. Presence, then, would rely on such characteristics of the situation as the embodied experience and the environment's responsiveness, which is closer to engagement than immersion. AR journalism would serve well in providing a new perspective in familiar surroundings, as many players of Pokémon Go, for example, have reported to have noticed. This is quite a different road than much of the VR applications have taken, and points towards approaches complementary to VR journalism, focusing on the local and specific sites. It may well be that there are lessons to be learned in how AR will be utilized in journalism, and how applications using AR will affect audience expectations and perceptions.

Finally, perhaps the most important lesson that game studies have for journalism is to avoid the immersive fallacy, or at least become aware of its dangers. Focusing on feeling the "reality" of the events, or the feeling of "being there" (de la Peña et al. 2010) is but one way of approaching the question of immersion (or presence). Immersion may be a part of experiencing mediated environments, but it is only 
a part of that experience and, depending on the context, not necessarily the most important one. Instead, it can be used strategically, either to pull a user into an experience or push them out of it, when it is more useful, for example, that they should be reflecting on their experience. There is no one way to be immersed, so choosing what type of immersion to aim for and when is a design choice when building immersive experiences. In contexts like journalism, reflection may sometimes be more valuable than immersion.

\section{References}

Apter, Michael J. 1991. “A structural-phenomenology of play.” In: J.H. Kerr \& M.J. Apter (eds), Adult Play: A Reversal Theory Approach. Amsterdam: Swets \& Zeitlinger.

Archer, D. \& K. Finger. 2018. "Walking in another's virtual shoes: Do 360-degree video news stories generate empathy in viewers?" Academic Commons, Tow Center for Digital Journalism, Columbia University. https://academiccommons.columbia.edu/doi/ 10.7916/D8669W5C [Accessed 28 May 2019].

Calleja, G. 2011. "Incorporation: A renewed understanding of presence and immersion in digital games." In: DiGRA 2011: Think Design Play, 2011. Netherlands: Utrecht School of the Arts.

Csikszentmihalyi, M. \& I. Csikszentmihalyi. 1975. Beyond Boredom and Anxiety: The Experience of Play in Work and Games. San Francisco, CA: Jossey-Bass Publishers.

de la Peña, N., P.Weil, J. Llobera, E. Giannopoulos, A. Pomes, B. Spanlang, D. Friedman, M.V. Sanchez-Vives, \& M. Slater. 2010. "Immersive journalism: Immersive virtual reality for the first-person experience of news." Presence 19(4): 291-301.

Ermi, L. \& F. Mäyrä. 2005. "Fundamental components of the gameplay experience: Analysing immersion.” In: DiGRA 2005 Conference: Changing Views - Worlds in Play, 2005. Netherlands: Utrecht School of the Arts. www.digra.org/dl/db/06276.41516.pdf [Accessed 15 March 2020].

Financial Times. 2017. “The Uber game.” https://ig.ft.com/uber-game/ [Accessed 15 March 2020].

Herrera, F., J. Bailenson, E. Weisz, E. Ogle, \& J. Zaki. 2018. "Building long-term empathy: A large-scale comparison of traditional and virtual reality perspective-taking." PLOS ONE 13(10): e0204494. https://doi.org/10.1371/journal.pone.0204494.

Huizinga, Johan. 1971[1938]. Homo Ludens: A Study of the Play-Element in Culture. Boston: Beacon Press.

Hunger in L.A. [Hunger in Los Angeles]. 2012.VR documentary by Nonny de la Peña. https:// emblematicgroup.com/experiences/hunger-in-la/ [Accessed 15 March 2020].

iNK Stories. 2016. 1979 Revolution: Black Friday. New York: iNK Stories.

Kent, T. 2015. "An ethical reality check for virtual reality journalism.” https://medium. com/@tjrkent/an-ethical-reality-check-for-virtual-reality-journalism-8e5230673507 [Accessed 15 March 2020].

Kool, H. 2016. "The ethics of immersive journalism: A rhetorical analysis of news storytelling with virtual reality technology." Intersect: The Stanford Journal of Science, Technology, and Society 9(3): 1-11.

Mäyrä, Frans. 2008. Introduction to Game Studies: Games in Culture. London; New York: SAGE. McMahan, A. 2003. "Immersion, engagement and presence." In: M.J.P. Wolf \& B. Perron (eds), The Video Game Theory Reader. New York: Routledge, p. 86. 
Murray, Janet H. 1997. Hamlet on the Holodeck: The Future of Narrative in Cyberspace. New York: Free Press.

Newman, James. 2004. Videogames. London: Routledge.

Ryan, Marie-Laure. 2001. Narrative as Virtual Reality: Immersion and Interactivity in Literature and Interactive Media. Baltimore; London: The Johns Hopkins University Press.

Salen, K. \& E. Zimmerman. 2004. Rules of Play: Game Design Fundamentals. Cambridge, MA: MIT Press.

Stenros, J. 2014. "In defence of a magic circle: The social, mental and cultural boundaries of play." Transactions of the Digital Games Research Association 1(2): 147-185.

Yee, Nicholas. 2002. “Ariadne: Understanding MMORPG addiction.” Available online: www.nickyee.com/hub/addiction/addiction.pdf [Accessed 15 March 2020]. 